\title{
Obesity does not aggravate osteoporosis or osteoblastic insulin resistance in orchiectomized rats
}

\author{
Saranyapin Potikanond ${ }^{1,2}$, Pinyada Rattanachote', Hiranya Pintana ${ }^{2}$, \\ Panan Suntornsaratoon $^{3,4}$, Narattaphol Charoenphandhu ${ }^{3,4}$, Nipon Chattipakorn ${ }^{2,5,6}$ \\ and Siriporn Chattipakorn ${ }^{2,7}$
}

'Department of Pharmacology, Faculty of Medicine, Chiang Mai University, Chiang Mai, Thailand ${ }^{2}$ Neurophysiology Unit, Cardiac Electrophysiology Research and Training Center, Faculty of Medicine, Chiang Mai University, Chiang Mai, Thailand

${ }^{3}$ Center of Calcium and Bone Research (COCAB), Faculty of Science, Mahidol University, Bangkok, Thailand ${ }^{4}$ Department of Physiology, Faculty of Science, Mahidol University, Bangkok, Thailand

${ }^{5}$ Cardiac Electrophysiology Unit, Department of Physiology, Faculty of Medicine, Chiang Mai University, Chiang Mai, Thailand

${ }^{6}$ Center of Excellence in Cardiac Electrophysiology Research, Chiang Mai University, Chiang Mai, Thailand ${ }^{7}$ Department of Oral Biology and Diagnostic Sciences, Faculty of Dentistry, Chiang Mai University, Chiang Mai, Thailand

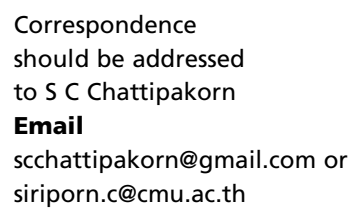

\begin{abstract}
The present study aimed to test the hypothesis that testosterone deprivation impairs osteoblastic insulin signaling, decreases osteoblast survival, reduces bone density, and that obesity aggravates those deleterious effects in testosterone-deprived rats. Twenty four male Wistar rats underwent either a bilateral orchiectomy $(O, n=12)$ or a sham operation $(S, n=12)$. Then the rats in each group were further divided into two subgroups fed with either a normal diet (ND) or a high-fat diet (HF) for 12 weeks. At the end of the protocol, blood samples were collected to determine metabolic parameters and osteocalcin ratios. The tibiae were collected to determine bone mass using microcomputed tomography and for osteoblast isolation. The results showed that rats fed with HF (sham-operated HF-fed rats (HFS) and ORX HF-fed rats (HFO)) developed peripheral insulin resistance and had decreased trabecular bone density. In ND-fed rats, only the ORX ND-fed rats (NDO) group had decreased trabecular bone density. In addition, osteoblastic insulin resistance, as indicated by a decrease in tyrosine phosphorylation of the insulin receptor and Akt, were observed in all groups except the sham-operated ND-fed rats (NDS) rats. Those groups, again with the exception of the NDS rats, also had decreased osteoblastic survival. No differences in the levels of osteoblastic insulin resistance and osteoblastic survival were found among the NDO, HFS, and HFO groups. These findings suggest that either testosterone deprivation or obesity alone can impair osteoblastic insulin signaling and decrease osteoblastic survival leading to the development of osteoporosis. However, obesity does not aggravate those deleterious effects in the bone of testosterone-deprived rats.
\end{abstract}

\section{Key Words}

- osteoblastic insulin signaling

- testosterone deprivation

- high-fat diet induced obesity
Journal of Endocrinology (2016) 228, 85-95
(C) 2016 Society for Endocrinology Printed in Great Britain
Published by Bioscientifica Ltd. 


\section{Introduction}

Insulin and insulin receptors play important roles in bone development and in promoting bone formation (Pun et al. 1989, Fulzele et al. 2010). Several studies have demonstrated that the downstream signaling molecules of insulin receptors, including IR, IRS1 and Akt, are required for osteoblastic proliferation and differentiation (Ogata et al. 2000, Kawamura et al. 2007). Our previous study demonstrated that obese-insulin resistant rats induced by a 12-week high-fat diet (HF) resulted in impairment of osteoblastic insulin signaling, decreased osteoblastic proliferation, increased osteoblastic apoptosis, and can lead to osteoporosis in the jaw bone (Pramojanee et al. 2013).

Preclinical and clinical studies have reported that obesity is associated with low serum testosterone levels or testosterone deficiency (Wang et al. 2011, Saboor Aftab et al. 2013). In addition, studies indicate that a state of testosterone deficiency could impair glucose homeostasis and insulin signaling, leading to the development of obesity, insulin resistance, and type 2 diabetes mellitus (Grossmann et al. 2009). Several studies have also demonstrated that a testosterone-deficient condition causes osteopenia and osteoporosis (Dupree \& Dobs 2004, Tsujimura 2013). However, the effects of combined testosterone deprivation and obese insulin resistance on bone morphology, osteoblastic insulin signaling and cell survival have not yet been investigated. The present study aimed to determine the effect of combined testosterone deprivation plus an obese-insulin resistant condition on bone morphology, osteoblastic insulin signaling, and osteoblastic survival. The hypotheses of the present study are that i) either testosterone deprivation alone or an obeseinsulin resistant condition alone causes impairment of osteoblastic insulin signaling, a decrease in osteoblastic survival and leads to osteoporosis, or ii) an obesity-induced insulin resistant condition aggravates those deleterious effects on bone under testosterone-deprived conditions.

\section{Materials and methods}

\section{Animal preparation}

All experiments were conducted in accordance with the approved protocol of the Faculty of Medicine, Chiang Mai University and the Institutional Animal Care Committee. Twenty-four male Wistar rats weighing 180-200 g were purchased from the National Animal Center, Salaya Campus, Mahidol University, Bangkok, Thailand.

\section{Experimental protocol}

The rats were divided into two groups ( $n=12 /$ group), either the sham-operation (S) group or the bilateral orchiectomy $(\mathrm{O})$ group. After 1 week following the orchiectomy, the rats in each group were further divided into two subgroups to receive either a normal diet (ND) or a $\mathrm{HF}$ for an additional 12 weeks ( $n=6 /$ sub-group), following the procedure decribed in a study by Pramojanee et al. (2013). Blood samples were collected from the rats' tails for determination of glucose, cholesterol, insulin, testosterone, and osteocalcin levels at week 0 and week 12 . At the end of the experiment, animals were deeply anesthetized and sacrificed. The tibiae were removed, cleaned, and kept with in normal saline at $-80^{\circ} \mathrm{C}$ before micro-computed tomography (micro-CT) and histomorphometry analysis. Also, total visceral fat, including peritoneal, periovarian, and perirenal fat pads were removed and weighed (Pratchayasakul et al. 2014). A summary of the experimental protocol is provided in Fig. 1.

\section{Orchiectomy procedure}

Orchiectomy was performed using the method described in previous studies (Dulisch 1976, Pongkan et al. 2015).

\section{Plasma analysis}

Plasma glucose and cholesterol levels were determined by a colorimetric assay (Biotech, Bangkok, Thailand). Plasma HDL and LDL levels were determined using a commercial colorimetric assay kit (Biovision, CA, USA). Plasma insulin levels were determined using a Sandwich ELISA kit (LINCO Research, St. Claire, MO, USA). Plasma testosterone levels were measured using the electrochemiluminescence immunoassay 'ECLIA' technique (Roche Diagnostic). Peripheral insulin resistance was assessed by the homeostasis model assessment (HOMA) (Haffner et al. 1997,

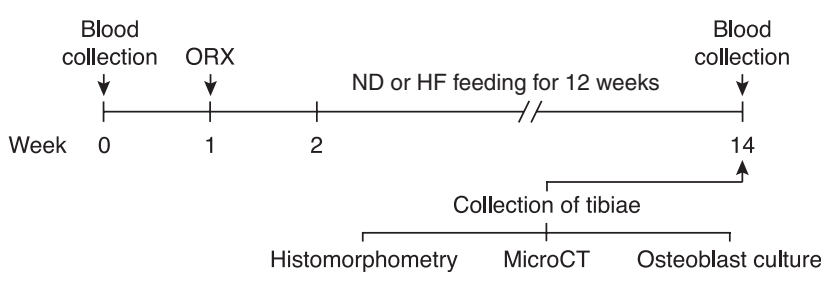

Figure 1

Experimental schematic. At the start of the first week, rats were divided into two groups: a sham-operation (S) group and a bilateral orchiectomy $(\mathrm{ORX})$ group. One week after surgery, rats in each group were divided into two subgroups, receiving either a ND or a HF for 12 weeks.

Published by Bioscientifica Ltd. 
Appleton et al. 2005), a mathematical model describing the degree of insulin resistance, calculated from fasting plasma insulin and fasting plasma glucose concentration. A higher HOMA index indicates a higher degree of insulin resistance. The HOMA index was determined by the following equation: (fasting plasma insulin $(\mu \mathrm{U} / \mathrm{ml})) \times$ (fasting plasma glucose $(\mathrm{mmol} / \mathrm{l})) / 22.5$ (Haffner et al. 1997, Appleton et al. 2005). The plasma Gla and Glu osteoclacin levels were measured by a rat Glu-Osteocalcin high sensitive EIA Kit (Takara Bio, Inc., Shiga, Japan) with $0.125 \mathrm{ng} / \mathrm{ml}$ minimum sensitivity of detection, $4.8 \%$ inter assay, and $6.3 \%$ intra assay.

\section{Primary osteoblastic culture}

Left tibiae were prepared in pieces for osteoblastic isolation using the serial enzymatic method (Pramojanee et al. 2013). Osteoblastic cells were identified with Alizarin red (Sigma-Aldrich) and alkaline phostphatase (Wako, Osaka, Japan), as described in a previous study (Pramojanee et al. 2013).

\section{Cell proliferation and apoptosis assay}

The osteoblastic cells were plated on glass coverslips in 96-well plates at a density of $1 \times 10^{4}$ cells/well and cultured for $24 \mathrm{~h}$ in DMEM which was supplemented with 10\% FBS as previously described (Pramojanee et al. 2013). Cell proliferation and apoptosis assay was carried out by the following method: the osteoblasts isolated from all groups were plated in 96-well plates at the density of $1 \times$ $10^{4}$ cells/well and cultured in DMEM supplemented with $10 \%$ FBS for $24 \mathrm{~h}$. Cells were starved by DMEM supplemented with $1 \%$ FBS for $24 \mathrm{~h}$ before stimulating with $10 \mathrm{nM}$ insulin for $24 \mathrm{~h}$. Cell proliferation levels were measured by a AlamarblueTM fluorometric cell viability assay (Biosource, California, USA). A TUNEL assay (ClickiT TUNEL Alexa Fluor imaging assays; Invitrogen) was used to determine cellular apoptosis levels, as described in a previous study: (Pramojanee et al. 2013). Cell proliferation/apoptosis was given as a percentage of the control (non-insulin stimulated cells). Three sections per sample were used in the TUNEL analysis.

\section{Western blot analysis}

The osteoblastic cells were lysed in a lysis buffer (20 mmol/l Tris-HCl, $1 \mathrm{mmol} / \mathrm{l} \mathrm{Na} \mathrm{VO}_{4}, 5 \mathrm{mmol} / \mathrm{l} \mathrm{NaF}$ and phosphatase inhibitor) containing protease inhibitors. Protein analysis using western blot analysis was performed as previously decribed (Pramojanee et al.
2013). The protein preparations were separated by $10 \%$ w/v SDS-PAGE and transferred to PVDF membranes (Millipore, MA, USA). Primary antibodies at 1:1000 were used for phosphorylation of IR (Tyr 1162/1163), IRBsubunit and Cyclin D1 (Santa Cruz Biotechnology, Inc.). $\beta$-actin at 1:2000 (Santa Cruz Biotechnology, Inc.) was used as a loading control. pAkt (Ser 473), Akt and secondary at 1: 1000, anti-rabbit or anti-mouse HRP conjugated antibodies at 1:2000 were obtained from Cell Signaling Technology, Inc. (Danvers, MA, USA). The protein bands were visualized by ECL western blotting detection reagent and quantitated using ImageJ Software (NIH, Bethesda, MA, USA; http://imagej.nih.gov/ij/).

\section{Micro-CT and trabecular microstructural analysis}

Right tibiae were analyzed by a micro-CT system (model SkyScan 1178;SkyScan, Kontich, Belgium) followed by hisomorphomety for trabecular microstructural analysis as previously described (Suntornsaratoon et al. 2014). We analyzed the results by using a section in the mid-sagittal plane, and examined 25 areas within the tibial secondary spongiosa.

\section{Statistical analysis}

All data were expressed as mean \pm s.E.M. One-way ANOVA with Dunnett's multiple comparison test was used for multiple sets of data. $P<0.05$ was considered statistically significant.

\section{Results}

HF consumption, but not testosterone deprivation, caused peripheral insulin resistance

At baseline levels (before orchiectomy), no metabolic parameters were significantly different between rats in the sham-operation (S) group and rats in the orchiectomized group (Table 1). The success of the orichectomy was

Table 1 Metabolic parameters at baseline (week 0) before operating on the sham-operated group (SHAM) and the orchiectomized group (ORX)

Metabolic parameters
Body weight $(\mathrm{g})$
Plasma glucose $(\mathrm{mg} / \mathrm{dl})$
Plasma insulin $(\mathrm{ng} / \mathrm{ml})$
HOMA index
Plasma total cholesterol $(\mathrm{mg} / \mathrm{dl})$

\begin{tabular}{rrr}
\multicolumn{1}{c}{ SHAM } & \multicolumn{1}{c}{ ORX } \\
\cline { 1 - 1 } $191.67 \pm 3.33$ & & \multicolumn{1}{c}{$197.50 \pm 4.03$} \\
$105.64 \pm 6.49$ & & $109.18 \pm 4.54$ \\
$2.31 \pm 0.33$ & & $2.33 \pm 0.31$ \\
$14.02 \pm 1.19$ & & $14.98 \pm 1.98$ \\
$48.33 \pm 3.69$ & & $47.72 \pm 2.53$
\end{tabular}

Published by Bioscientifica Ltd 
Table 2 Metabolic parameters and plasma osteoclacin levels following either orchiectomy or sham operation

\begin{tabular}{lcc} 
Metabolic parameters & NDS \\
\cline { 1 - 1 } Body weight $(\mathrm{g})$ & & $475 \pm 8.5$ \\
Visceral fat $(\mathrm{g})$ & & $31.8 \pm 2.8$ \\
Plasma insulin (ng/ml) & $1.7 \pm 0.2$ \\
Plasma glucose (mg/dl) & $145.2 \pm 6.0$ \\
HOMA index & $14.6 \pm 1.7$ \\
Plasma total cholesterol (mg/dl) & $49.4 \pm 3.7$ \\
Plasma testosterone (ng/dl) & & $0.75 \pm 0.2$ \\
Gla osteoclacin (ng/ml) & $150.3 \pm 11.6$ \\
Glu osteoclacin (ng/ml) & $39.2 \pm 3.5$ \\
Osteocalcin Gla/Glu ratio & $4.5 \pm 0.4$ \\
\hline
\end{tabular}

\begin{tabular}{c}
\hline NDO \\
\hline $425.8 \pm 10.8^{*, \dagger}$ \\
$18.8 \pm 3.7^{*, \dagger}$ \\
$2.0 \pm 0.3$ \\
$143.2 \pm 12.5$ \\
$17.2 \pm 3.2$ \\
$50.0 \pm 5.7$ \\
$0.07 \pm 0.01^{*, \dagger}$ \\
$171.9 \pm 13.1$ \\
$51.8 \pm 3.9^{*}$ \\
$3.3 \pm 0.2^{*}$
\end{tabular}

\begin{tabular}{c}
\hline HFS \\
\hline $550 \pm 16.9^{*}$ \\
$46.0 \pm 4.5^{*}$ \\
$3.1 \pm 0.4^{*}$ \\
$147.4 \pm 6.5$ \\
$26.6 \pm 3.0^{*}$ \\
$70.7 \pm 3.3^{*}$ \\
$0.46 \pm 0.01^{*}$ \\
$171.7 \pm 14.0$ \\
$55.4 \pm 5.6^{*}$ \\
$3.3 \pm 0.3^{*}$ \\
\hline
\end{tabular}

\begin{tabular}{c}
\hline HFO \\
\hline $510 \pm 14.1^{\dagger}$ \\
$30.7 \pm 2.8^{\dagger}$ \\
$2.9 \pm 0.3^{*}$ \\
$146.3 \pm 7.3$ \\
$25.2 \pm 4.1^{*}$ \\
$65.3 \pm 7.0^{*}$ \\
$0.15 \pm 0.06^{*}+$ \\
$163.8 \pm 30.0$ \\
$55.2 \pm 8.6^{*}$ \\
$3.8 \pm 0.3^{*}$ \\
\hline
\end{tabular}

${ }^{*} P<0.05$ vs NDS, ${ }^{\dagger} P<0.05$ vs HFS.

confirmed by decreased plasma testosterone levels (Table 2). Interestingly, the testosterone levels of the sham-operated HF-fed rats (HFS) group also decreased significantly compared with that of the sham-operated ND-fed rats (NDS) group. After 12 weeks of the dietry protocol, both the HFS and the ORX HF-fed rats (HFO) rats presented characteristics of impaired peripheral insulin sensitivity as indicated by increased body weight, increased visceral fat, hyperinsulinemia with euglycemia, an increased HOMA index and increased plasma cholesterol levels when compared with the NDS group (Table 2). Although the body weight and quanity of visceral fat in the HFO group were significantly greater than in the NDS group, the HFO group had a lower body weight and less visceral fat than the HFS group (Table 2). In addition, the ORX ND-fed rats (NDO) rats had significantly decreased body weight and visceral fat compared with the NDS group. These findings suggest that a HF, but not testosterone deprivation, caused the obesity and the peripheral insulin resistance.

\section{Decreased Gla/Glu osteocalcin ratio observed in both HF-fed rats and testosterone-deprived rats}

The plasma Gla/Glu-osteocalcin ratio was used as a marker of bone formation. It was found that the Gla/Glu osteocalcin ratio was significantly decreased in the NDO, HFS, and HFO groups compared with that of the NDS group (Table 2). However, the ratios were not different among the NDO, HFS, and HFO rats. These findings suggest that either HF consumption or testosterone deprivation can cause a reduction in bone formation. Obesity, however, did not aggravate this deleterious effect in testosterone-deprived rats.
Trabecular bone microstructure deceased in HF-fed rats and in testosterone-deprived rats

The morphology of the tibial bones was analysed using a micro-CT. The cortical parameters, inclucing $x$-axis moment of inertia, $y$-axis moment of inertia, cortical thickness, cortical bone area, cortical periosteal perimeter, and cortical endosteal perimeter were not different among the groups (Fig. 2A, B, C, D, and F). Interestingly, trabecular bone mineral density was significantly decreased in the NDO, HFS, and HFO groups (Fig. 2G). This indicated thatosteoporosis occurred in this area. Bone histomorphometry was used to further investigate the bone microstructure. Goldner's trichrome-stained tibial sections indicated that testosterone deprivation and HF consumption led to a decrease in mineralized tissue (green) and bone volume (Fig. 3A and B). Testosterone deprivation in both $\mathrm{ND}$ and $\mathrm{HF}$ rats increased trabecular separation (Fig. 3D) and decreased trabecular number (Fig. 3E). The decrease in trabecular bone volume in HFS was probably the result of the decreased trabecular number rather than the decreased trabecular thickness (Fig. 3E).

\section{Osteoblastic proliferation significantly decreased in HF-fed rats and testosterone-deprived rats}

Cells isolated from tibiae and cultured in osteogenic medium were positively stained with Alizarin red (Fig. 4A, C, E, and G) and alkaline phosphatase (Fig. 4B, D, F, and H), to identify osteoblastic characteristics. AlamarBlue fluoremetric cell viability assay was performed at 1, 2, 4, 6, 24, 48, and $72 \mathrm{~h}$ after initial culturing. The number of cells was significantly decreased in the NDO, HFS, and HFO groups at 48 and $72 \mathrm{~h}$ when compared with the NDS group (Fig. 4J).

Published by Bioscientifica Ltd. 

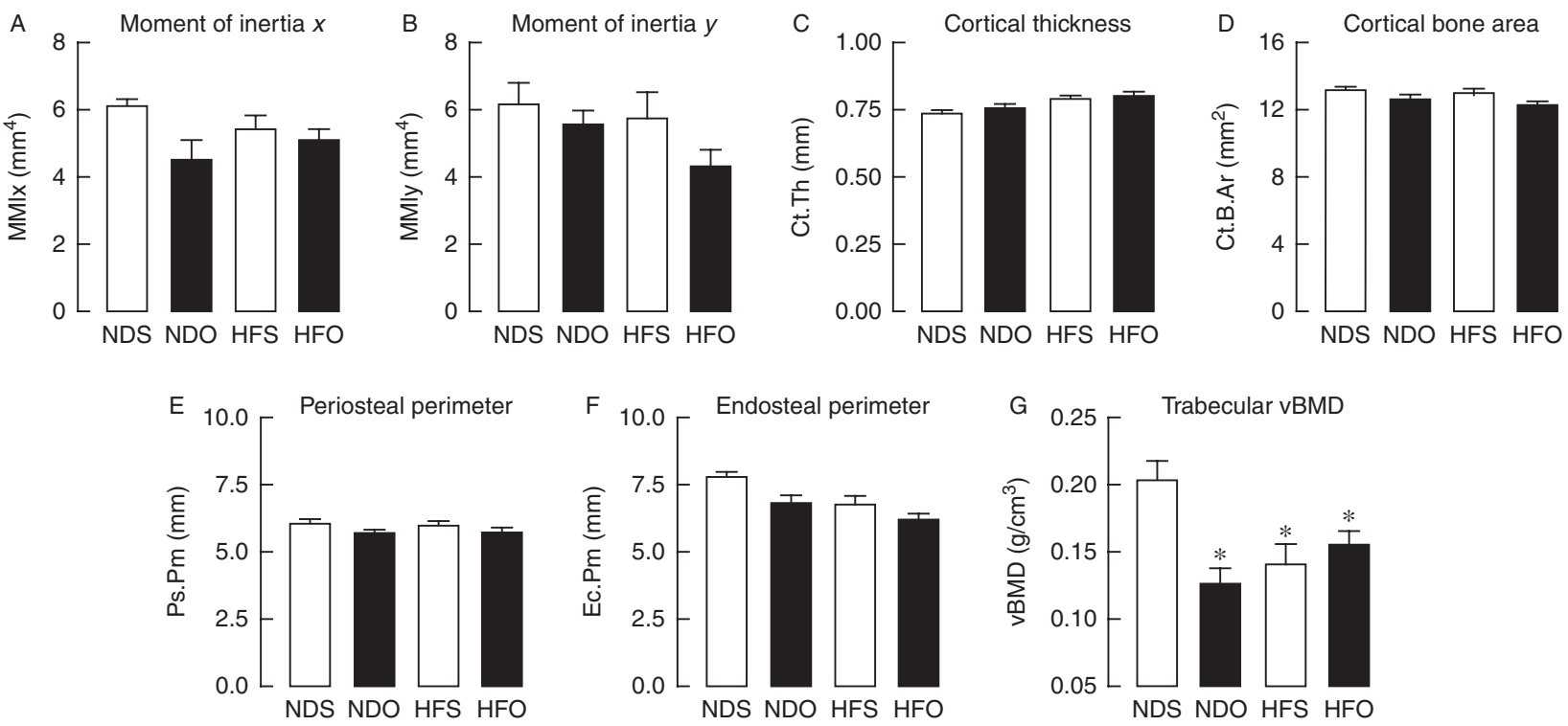

Figure 2

The tibial diaphysis of rats at 12 weeks post surgery was determined by micro-CT, $x$-axis moment of inertia (MMIx; A), $y$-axis moment of inertia (MMly; B), cortical thickness (Ct.Th; C), cortical bone area (Ct.B.Ar; D),

A proliferation signal, Cyclin D1 protein, was decreased in the HF-fed rats and testosterone-deprived rats (Fig. 4L). No differences in these factors were found among the NDO, HFS, and HFO groups. These findings suggest that either cortical periosteal perimeter (Ct.Pe.Pm; E), cortical endosteal perimeter (Ct.En.Pm; F), and tibial bone mineral density (T.BMD; G). ${ }^{\star} P<0.05$ vs NDS group.

testoterone deprivation alone or HF consumption alone can cause a reduction in osteoblastic proliferation, but that obesity induced by HF consumption does not aggravate this effect in testosterone-deprived rats.
A

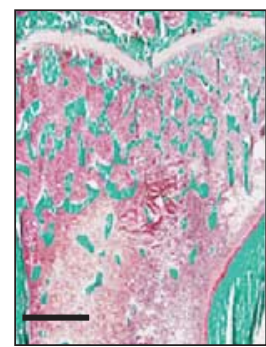

NDO

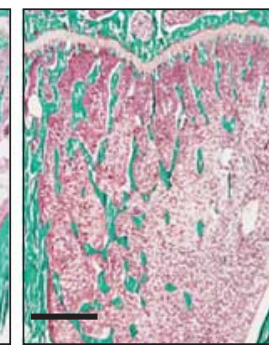

HFS

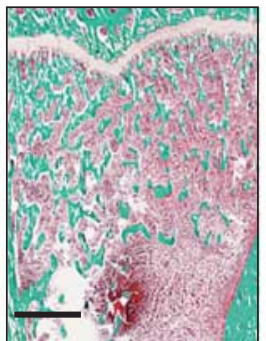

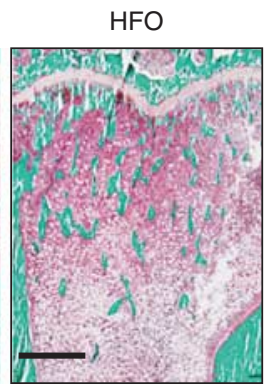

B

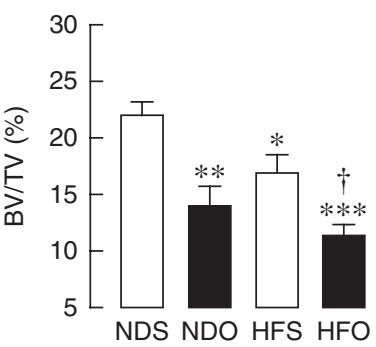

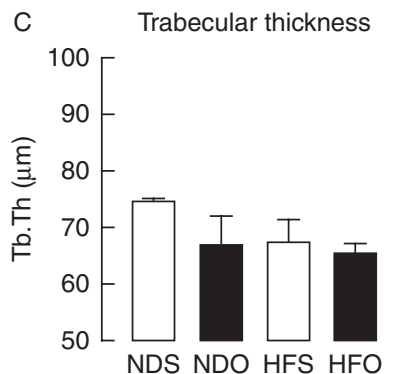

Figure 3

Micrographs of proximal tibial metaphyses (A). Scale bars $=1000 \mu \mathrm{m}$. Bar graphs represent bone volume fraction (BV/TV) (B), trabecular thickness (Tb.Th) (C), trabecular separation (Tb.Sp) (D), and trabecular number
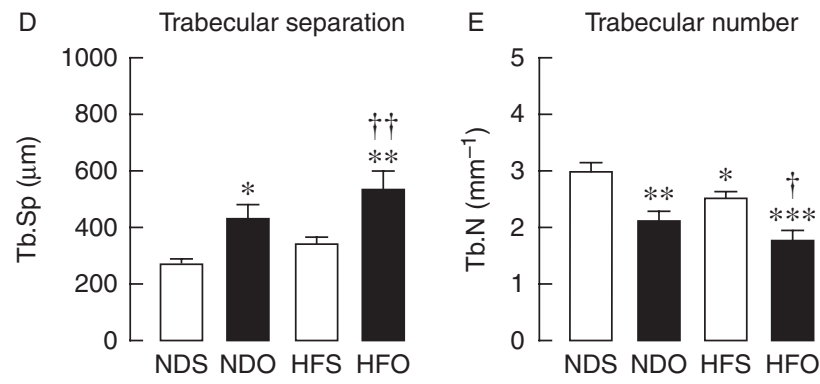

(Tb.N) (E). ${ }^{*} P<0.05$ vs NDS group; $* * P<0.01$ vs NDS group; $* * * P<0.001$ vs the NDS group; ${ }^{\dagger} P<0.05$ vs the HFS group; ${ }^{\dagger+} P<0.01$ vs the HFS group. A full colour version of this figure is available at http://dx.doi.org/10.1530/JOE-15-0333. 

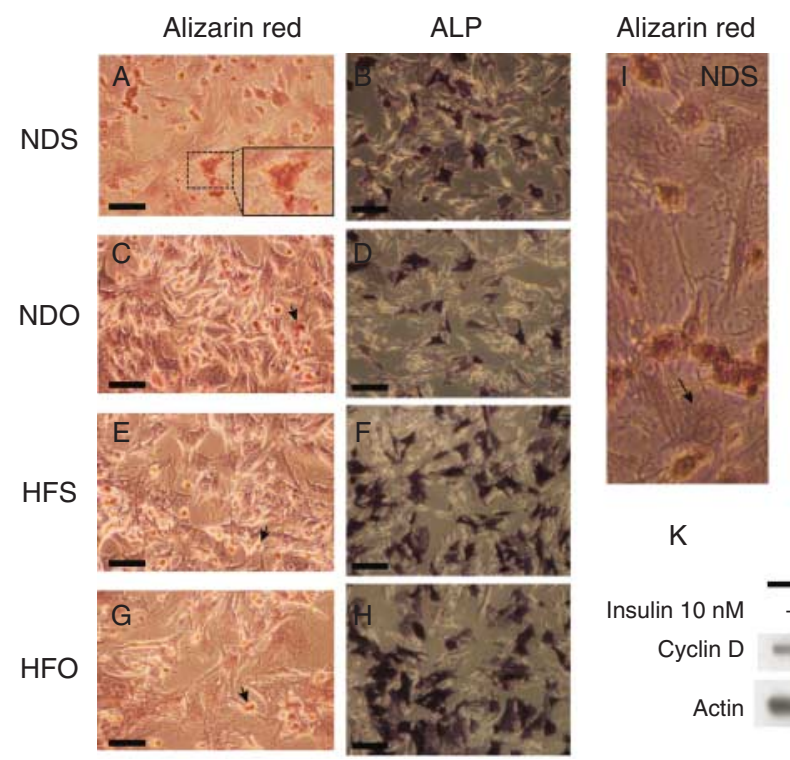

$\mathrm{J}$

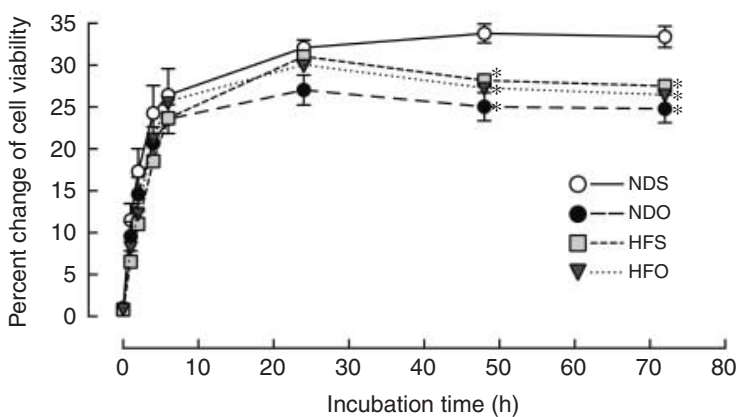

K
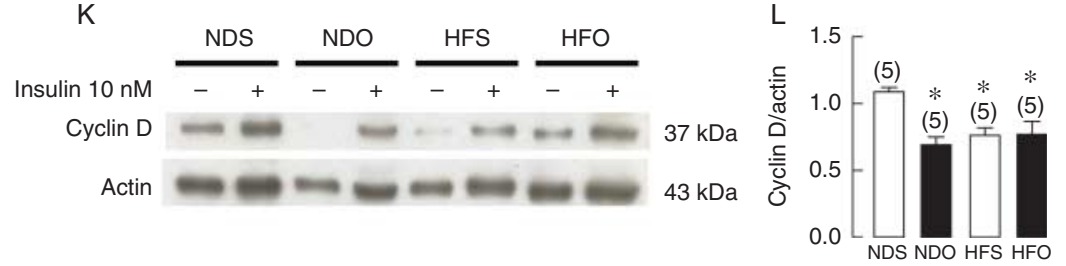

\section{Figure 4}

Alizarin red $(A, C, E$, and $G)$ and alkaline phosphatase staining $(B, D, F$, and $\mathrm{H}$ ) were performed to characterize osteoblasts. (I) Magnified image of cultured osteoblasts (stellate cell with nucleus; arrow) from NDS group. Alizarin-stained nodules (red) are scattered in matrix. The percentage change of cell viability using alamarBlue fluoremetric cell viability assay is shown in J. The representation of cyclinD protein expression with insulin

\section{Osteoblastic apoptosis significantly increased in both HF-fed rats and testosterone-deprived rats}

Apoptotic cell death, measured with TUNEL assay, significantly increased in the NDO, HFS, and HFO groups when compared with the NDS group (Fig. 5). No differences in apoptotic cell death were found among the NDO, HFS, and HFO groups. These findings suggest that either obesity alone or testosterone deprivation alone results in increased osteoblastic apoptosis. However, obesity induced by HF consumption did not aggravate apoptotic cell death in testosterone-deprived rats.

\section{Osteoblastic insulin signaling impaired in both HF-fed rats and testosterone-deprived rats}

The osteoblastic insulin signaling activity was determined by tyrosine phosphorylation of insulin receptors after stimulation with10 $\mathrm{nM}$ of insulin for $10 \mathrm{~min}$. The phosphorylation of the insulin receptor was decreased in the NDO, HFS, and HFO groups compared with that of the NDS group (Fig. 6A and B). In addition, the phosphorylation of the downstream insulin signaling molecule, Akt, was significantly decreased in the NDO, stimulation $(+)$ and without insulin stimulation $(-)$ are shown in $\mathrm{K}$. Bar graphs represent densitometric values of cyclin $D$ calculating by the level of cyclin $D$ without insulin stimulation normalizing with level of actin (L). ${ }^{*} P<0.05$ vs NDS group. A full colour version of this figure is available at $\mathrm{http}: / / \mathrm{dx}$.doi.org/10.1530/JOE-15-0333.

HFS, and HFO groups when compared with that in the NDS group (Fig. 6A and C).

\section{Discussion}

Based on the findings of this study, it appears that HF consumption, not testosterone deprivation, causes obesity and peripheral insulin resistance. Furthermore, testosterone deprivation alone and HF-induced obsity alone lead to a decrease in serum osteocalcin, decreased osteoblastic proliferation, increased osteoblastic apoptosis, osteoblastic insulin resistance, and the impairment of bone microarchitectures. In addition, an obesity-induced insulin resistant condition does not aggravate those deleterious effects in the bones of testosterone-deprived rats.

The present findings and those of previous reports (Winzell \& Ahren 2004, Pramojanee et al. 2013) demonstrate that obesity induced by 12 -week HF consumption causes peripheral insulin resistance as indicated by increased body weight, increased visceral fat, hyperinsulinemia with euglycemia, and an increased HOMA index. The development of peripheral insulin resistance in the present study was dependent on HF-induced obesity, but not testosterone deprivation, as confirmed by the fact

Published by Bioscientifica Ltd. 

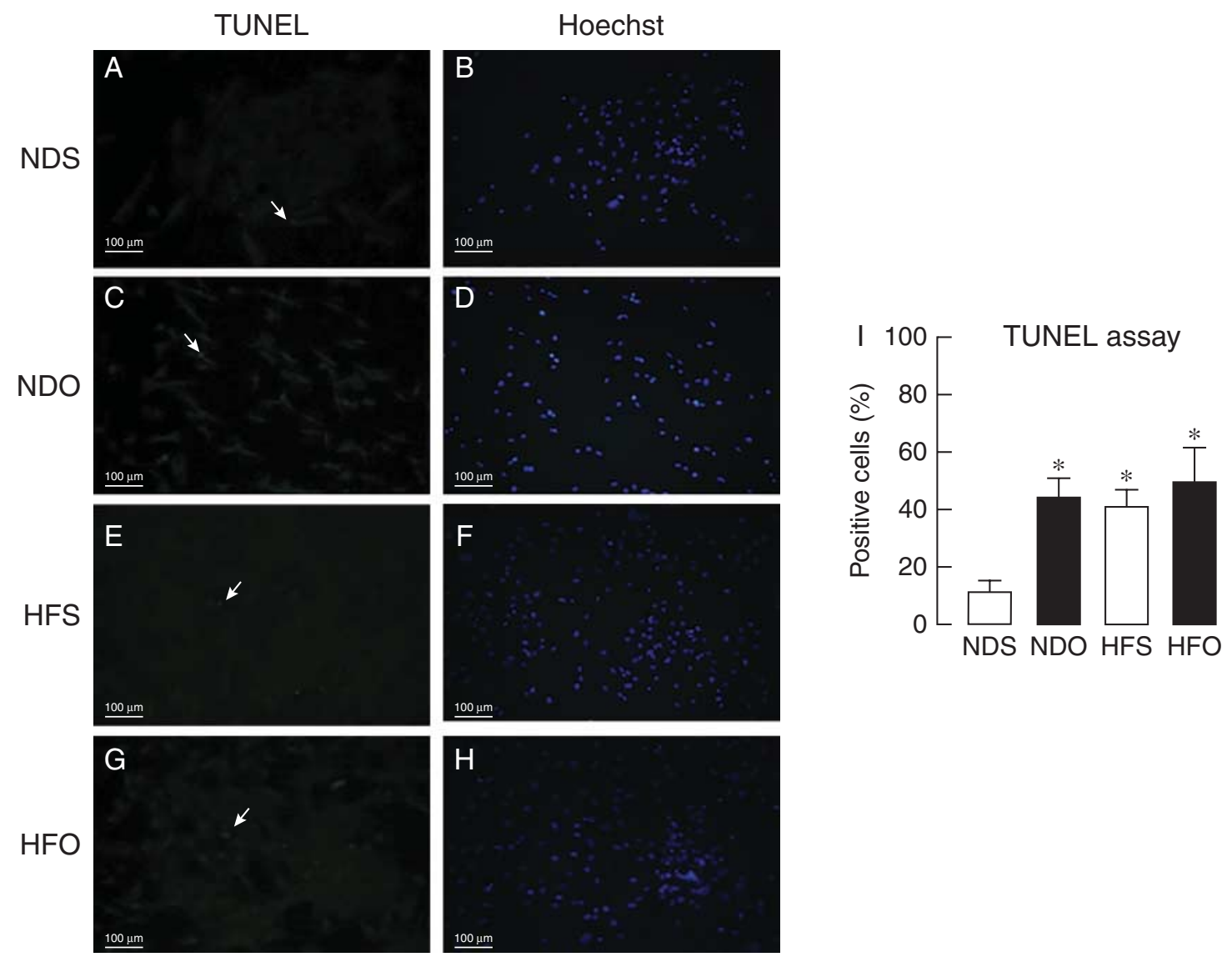

Figure 5

Measurement of apoptotic cell death was performed by TUNEL assay. Apoptotic cells were labeled with green fluorescent (A, C, E, and G) and are indicated by arrows. Nuclease labeling with Hoechst stain is shown in $B, D$,

that there was no change in the HOMA index in the NDO rats. These findings are consistent with a report by Christoffersen et al. (2006). However, several previous studies have reported that testosterone deprivation alone via orchiectomy (ORX) in male rodents causes impairment of insulin sensitivity (Xia et al. 2013, Jayaraman et al. 2014a). For example, Xia et al. (2013) found that ORX-induced testosterone deficiency increased fasting blood glucose in mature adult (10 week old) male Sprague-Dawley rats. Jayaraman et al. (2014a b) demonstrated that ORX adult mice (3 months of age) had impaired insulin sensitivity. Differences in the breed and age at the start of the ORX procedure (this study used young adult male Wistar rats orchiectomized at 5-6 weeks of age rather than Sprague-Dawley rats) might be the cause of the differences in the results.

Previous studies reported the role of osteocalcin in increased insulin sensitivity in the target organs (Saleem
$\mathrm{F}$, and $\mathrm{H}$. Scale bars are $100 \mu \mathrm{m}$. The bar graph represents the percentage of positive TUNEL cells (I). ${ }^{*}<0.05$ vs NDS group. A full colour version of this figure is available at http://dx.doi.org/10.1530/JOE-15-0333.

et al. 2010, Xiao et al. 2014). The present study showed that the ratio of Gla/Glu ratio and Glu osteocalcin levels in NDO, HFS, and HFO groups significantly reduced, when compared with that of the NDS group. However, Gla osteocalcin levels among all four groups were not significantly different. These findings suggested that the reduction of Gla/Glu osteocalcin ratio in NDO, HFS, and HFO groups was due to increased Glu levels, implying that decreased insulin sensitivity could occur in NDO, HFS, and HFO groups. The present study, however, also demonstrated a reduction in the Gla/Glu osteovlacin ratio as well as the impairment of osteoblastic insulin signaling as indicated by decreased phosphorylation of the insulin receptor and its downstream signaling molecule, Akt protein, in either orchiectomy alone or HF-induced obesity alone. Our findings suggested that the osteoblastic insulin resistance in both testosterone-deprived rats and obese-insulin resistant rats could possibly occur via the

Published by Bioscientifica Ltd 
A

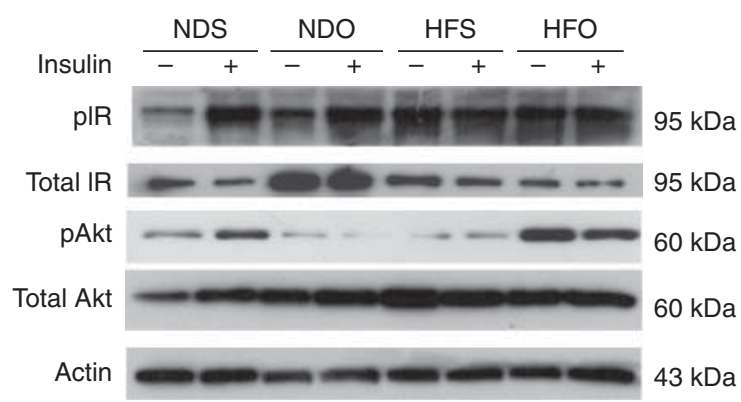

B

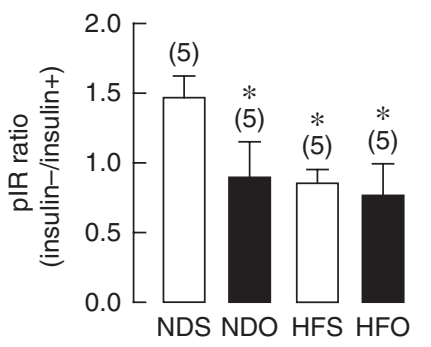

C

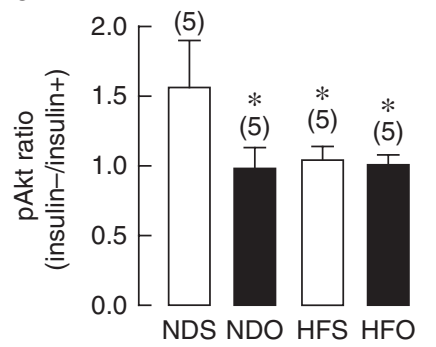

Figure 6

Effects of HFD and testosterone deprivation on insulin signaling. Cells were stimulated either with or without $10 \mathrm{nM}$ of insulin for $10 \mathrm{~min}$. Western blotting bands of pIR, total IR, pAkt, total Akt, and actin were shown in A. The bar graphs represent densitometric values of pIR (B) and pAkt (C) which

effects of low osteocalcin levels as previously observed in pancreatic $\beta$ cells and adipocytes (Ferron et al. 2008, Hwang et al. 2012).

The testosterone deprivation following orchiectomy was confirmed by the decreased testosterone level in the orchiectomized rats. However, the HFS rats also showed a decrease in plasma testosterone levels, a finding consistent with previous clinical reports which demonstrated that obesity in men is associated with low testosterone levels (Barrett-Connor 1992, Andersson et al. 1994, Grossmann 2013, Mogri et al. 2013). The reduction of testosterone levels in the HFS rats could be a factor in the decrease in the hypothalamo-pituitary-gonadal axis due to inflammation following obesity as decribed in previous studies (Cohen 1999, Gregor \& Hotamisligil 2011, Mammi et al. 2012, Jayaraman et al. 2014b). Although it has been shown that obesity could increase the estrogen levels in female rats (Pratchayasakul et al. 2011), our previous study demonstrated that 12 weeks of high-fat diet feeding did not affect the circulating estrogen levels in male rats (Pratchayasakul et al. 2011). Moreover, a recent report also demonstrated that estradiol administration did not affect cardiac function in orchiectomized male rats (Ayaz et al. 2015). All of these findings indicated the important role of decreased testosterone levels rather than increased estrogen levels in obese male rats.

A previous study demonstrated that orchiectomyinduced trabecular bone degeneration was possibly caused by a decrease in the expressions of osteogenic genes (Chin \& Ima-Nirwana 2015). Several studies have demonstrated that testosterone played an important role in osteoblastic proliferation by enhancing mitogenesis and differentiation in bone cells (Sömjen et al. 1989, Kasperk et al. 1990, Gray et al. 1992). In addition, testosterone has been are calculating by divided the insulin stimulation by without insulin stimulation (insulin +/insulin -), then normalizing with total protein expression. ${ }^{*} P<0.05$ vs NDS group.

shown to play a role in osteoblast apoptosis in which a lack of testosterone enhanced osteoblast apoptosis through an increased Bax/Bcl-2 ratio (Wiren et al. 2006, Chen et al. 2010). Our results showed that a reduction of the Gla/Glu osteocalcin ratio, decreased osteoblastic proliferation and increased osteoblastic apoptosis in testosterone-deprived rats was associated with microstructural changes in the tibial trabecular bones and a significant overall reduction in mineral density of those bones. Our findings and a previous report (Fulzele et al. 2010) demonstrated that the impairment of osteoblastic insulin receptors results in a decrease in osteoblast proliferation. All of these findings suggest that testosterone deprivation decreases osteoblastic activity and osteoblastic proliferation while increasing ostoblastic apoptosis, leading to osteoporosis.

The effects of obesity on bone density is still unclear. Several studies have shown that obesity could increase bone density (Harris et al. 1992, Albala et al. 1996, Vandewalle et al. 2013); however, other studies have found that obesity has either no effect on bone density (Greco et al. 2010) or increases bone porosity (Rosen \& Bouxsein 2006, Zhao et al. 2008, Gómez et al. 2009). The present study, also, found that obese and insulin resistant rats had a decreased Gla/Glu-osteocalcin ratio, decreased osteoblastic proliferation, increased osteoblastic apoptosis, osteoblastic insulin resistance and increased bone porosity. These findings indicate the deleterious effects of obesity on bone quality. Interestingly, obesity did not aggravate those forms of deterioration in testosteronedeprived rats as indicated by the lack of differences in trabecular bone quality between the HFO and the NDO groups (Fig. 3B, D, and E). There are several possible explanations for these findings. First, the deleterious effects found in osteoblasts of testosterone-deprived rats

Published by Bioscientifica Ltd 
could have been at their maximum level, thus obesity could not have had further additive effects on those osteoblastic cells. Secondly, it could be that the lack of testosterone, rather than obesity, that was the major aggressive factor causing the impairment of trabecular bone structures as this impairment was also observed in NDO rats. However, in the present study no significant differences in cortical bones were observed among the experimental groups. Two previous studies found that the cortical bone markers had markedly decreased 6 months after castration (Danielsen et al. 1992, Reim et al. 2008). It is possible that the 3-month period of castration in the present study might not have been long enough to observe cortical bone change. However, the hypothesis of this study is supported by the fact that the response of cortical bone mass to HF-induced obesity differs by age (IonovaMartin et al. 2011). The Ionova-Martin group demonstrated that femur thickness does not change in young mice after 16 weeks of HF comsumption although those mice, as in the mice in the present study, had poor bone quality.

The limitation of the present study was that testosterone deprivation and obesity were not independently assessed as the cause of osteoporosis due to the fact that decreased testosterone levels was also observed in the obese-insulin resistant rats. However, it has been shown previously that testosterone deprivation alone could cause osteoporosis (Tuck \& Francis 2009). Moreover, the recent study by Donner et al. (2015) demonstrated that dietinduced visceral obesity resulted in decreased bone mineral area and content and impaired femoral stiffness and strength without changes in testosterone levels. All of these findings indicated that either obesity or testosterone deprivation could lead to osteoporosis. In addition, the aim of the present study was to mainly investigate the synergistic effects of obesity and testosterone deprivation on osteoporosis. Findings from the present study clearly demonstrated that either obesity alone or testosterone deprivation alone led to osteoporosis, however no additive or synergistic effect of obesity and testosterone deprivation on osteoporosis could be found.

Lack of results pertaining to bone cellular activities is one of the limitations of the present study. However, neither serum bone biomarkers nor tissue samples for dynamic bone histomorphometry were collected from those rats. Therefore, it is not possible for us to quantify bone cellular activities in the present study. However, we believe that the available bone parameters provided in the present study provided sufficient evidence to confirm the detrimental effects of testosterone deprivation and insulin resistance on bone. The overall adiposity was not measured. However, the visceral fat was determined and used to represent the level of metabolic complications in this obese-insulin resistant model (Hansen et al. 1997, Morakinyo et al. 2015).

\section{Conslusions}

Either testosterone deprivation alone or HF-induced obesity alone can cause impairment of osteoblastic insulin signaling and reduction in osteoblastic survival, leading to osteoporosis. However, obesity does not augment the adverse effects on bone density under testosteronedeprived conditions.

\section{Declaration of interest}

The authors declare that there is no conflict of interest that could be perceived as prejudicing the impartiality of the research reported.

\section{Funding}

This study was supported by grants from the Thailand Research Fund (TRF-BRG5780016 to SC); TRF Senior Research Scholar (RTA5780001 to N Charoenphandhu), a NSTDA Research Chair Grant from the National Science and Technology Development Agency (NC) and a Chiang Mai University Excellent Center Award (NC) as well as the China Medical Board of New York; the Faculty of Medicine, Chiang Mai University (S P).

\section{Author contribution statement}

S P performed the experiments, analyzed the data, and wrote the manuscript. $P R$ performed the experiments and analyzed the data. $\mathrm{H} P$ performed the animal experiments and analyzed the metabolic data. $\mathrm{P} S$ and $\mathrm{Na} C$ performed and analyzed the bone histomophometry. $\mathrm{N} C$ designed the study and wrote the manuscript. S C designed the study, analyzed the data, and wrote the manuscript.

\section{Acknowledgements}

The authors would also like to thank Dr G Lamar Robert for assistance in editing the manuscript.

\section{References}

Albala C, Yáñez M, Devoto E, Sostin C, Zeballos L \& Santos JL 1996 Obesity as a protective factor for postmenopausal osteoporosis. International Journal of Obesity and Related Metabolic Disorders 20 1027-1032.

Andersson B, Marin P, Lissner L, Vermeulen A \& Bjorntorp P 1994 Testosterone concentrations in women and men with NIDDM. Diabetes Care 17 405-411. (doi:10.2337/diacare.17.5.405)

Appleton DJ, Rand JS \& Sunvold GD 2005 Basal plasma insulin and homeostasis model assessment (HOMA) are indicators of insulin sensitivity in cats. Journal of Feline Medicine and Surgery 7 183-193. (doi:10.1016/j.jfms.2004.12.002)

Published by Bioscientifica Ltd. 
Ayaz M, Akand M, Kucukbagriacik Y \& Dursunoglu D 2015 The effects of estradiol on cardiac muscle electrophysiology in orchiectomized rat model: a new insight to side effects caused by castration. European Review for Medical and Pharmacological Sciences 19 2866-2874.

Barrett-Connor E 1992 Lower endogenous androgen levels and dyslipidemia in men with non-insulin-dependent diabetes mellitus. Annals of Internal Medicine 117 807-811. (doi:10.7326/0003-4819-117-10-807)

Chen X, Deng Y, Zhou Z, Tao Q, Zhu J, Li X, Chen J \& Hou J 2010 17ßestradiol combined with testosterone promotes chicken osteoblast proliferation and differentiation by accelerating the cell cycle and inhibiting apoptosis in vitro. Veterinary Research Communications 34 143-152. (doi:10.1007/s11259-010-9340-2)

Chin K-Y \& Ima-Nirwana S 2015 The effects of orchidectomy and supraphysiological testosterone administration on trabecular bone structure and gene expression in rats. Aging Male 18 60-66. (doi:10.3109/13685538.2014.954995)

Christoffersen B, Raun K, Svendsen O, Fledelius C \& Golozoubova V 2006 Evalution of the castrated male Sprague-Dawley rat as a model of the metabolic syndrome and type 2 diabetes. International Journal of Obesity 30 1288-1297. (doi:10.1038/sj.ijo.0803261)

Cohen PG 1999 The hypogonadal-obesity cycle: role of aromatase in modulating the testosterone-estradiol shunt-a major factor in the genesis of morbid obesity. Medical Hypotheses 52 49-51. (doi:10.1054/ mehy.1997.0624)

Danielsen CC, Mosekilde L \& Andreassen TT 1992 Long-term effect of orchidectomy on cortical bone from rat femur: bone mass and mechanical properties. Calcified Tissue International 50 169-174. (doi:10.1007/BF00298796)

Donner DG, Elliott GE, Beck BR, Forwood MR \& Du-Toit EF 2015 The effects of visceral obesity and androgens on bone: trenbolone protects against loss of femoral bone mineral density and structural strength in viscerally obese and testosterone-deficient male rats. Osteoporosis International [in press]. (doi:10.1007/s00198-015-3345-1)

Dulisch ML 1976 A castration procedure for the rabbit, rat, hamster, and guinea pig. Journal of Zoo Animal Medicine 7 8-11. (doi:10.2307/ 20094380)

Dupree K \& Dobs A 2004 Osteopenia and male hypogonadism. Reviews in Urology 6 (Suppl 6) S30-S34.

Ferron M, Hinoi E, Karsenty G \& Ducy P 2008 Osteocalcin differentially regulates $\beta$ cell and adipocyte gene expression and affects the development of metabolic diseases in wild-type mice. PNAS 105 5266-5270. (doi:10.1073/pnas.0711119105)

Fulzele K, Riddle RC, DiGirolamo DJ, Cao X, Wan C, Chen D, Faugere M-C, Aja S, Hussain MA, Brüning JC et al. 2010 Insulin receptor signaling in osteoblasts regulates postnatal bone acquisition and body composition. Cell 142 309-319. (doi:10.1016/j.cell.2010.06.002)

Gómez JM, Vilarrasa N, Masdevall C, Pujol J, Solano E, Soler J, Elio I, Gallart L \& Vendrell J 2009 Regulation of bone mineral density in morbidly obese women: a cross-sectional study in two cohorts before and after bypass surgery. Obesity Surgery 19 345-350. (doi:10.1007/ s11695-008-9529-4)

Gray C, Colston KW, Mackay AG, Taylor ML \& Arnett TR 1992 Interaction of androgen and 1,25-dihydroxyvitamin D3: effects on normal rat bone cells. Journal of Bone and Mineral Research 7 41-46. (doi:10.1002/jbmr. 5650070107)

Greco EA, Fornari R, Rossi F, Santiemma V, Prossomariti G, Annoscia C, Aversa A, Brama M, Marini M, Donini LM et al. 2010 Is obesity protective for osteoporosis? Evaluation of bone mineral density in individuals with high body mass index International Journal of Clinical Practice 64 817-820. (doi:10.1111/j.1742-1241.2009.02301.x)

Gregor MF \& Hotamisligil GS 2011 Inflammatory mechanisms in obesity. Annual Review of Immunology 29 415-445. (doi:10.1146/annurevimmunol-031210-101322)

Grossmann M. 2013 Low Testosterone in Men with Type 2 Diabetes: Significance and Treatment.
Grossmann M, Panagiotopolous S, Sharpe K, MacIsaac RJ, Clarke S, Zajac JD, Jerums G \& Thomas MC 2009 Low testosterone and anaemia in men with type 2 diabetes. Clinical Endocrinology 70 547-553. (doi:10.1111/j. 1365-2265.2008.03357.x)

Haffner SM, Miettinen H \& Stern MP 1997 The homeostasis model in the San Antonio heart study. Diabetes Care 20 1087-1092. (doi:10.2337/ diacare.20.7.1087)

Hansen PA, Han DH, Nolte LA, Chen M \& Holloszy JO 1997 DHEA protects against visceral obesity and muscle insulin resistance in rats fed a highfat diet. American Journal of Physiology 273 R1704-R1708.

Harris S, Dallal GE \& Dawson-Hughes B 1992 Influence of body weight on rates of change in bone density of the spine, hip, and radius in postmenopausal women. Calcified Tissue International 50 19-23. (doi:10.1007/BF00297292)

Hwang Y-C, Jeong I-K, Ahn K-J \& Chung H-Y 2012 Circulating osteocalcin level is associated with improved glucose tolerance, insulin secretion and sensitivity independent of the plasma adiponectin level. Osteoporosis International 23 1337-1342. (doi:10.1007/s00198-011-1679-x)

Ionova-Martin SS, Wade JM, Tang S, Shahnazari M, Ager JW, Lane NE, Yao W, Alliston T, Vaisse C \& Ritchie RO 2011 Changes in cortical bone response to high-fat diet from adolescence to adulthood in mice. Osteoporosis International 22 2283-2293. (doi:10.1007/s00198-010-1432-x)

Jayaraman A, Lent-Schochet D \& Pike CJ 2014a Diet-induced obesity and low testosterone increase neuroinflammation and impair neural function. Journal of Neuroinflammation 11 162. (doi:10.1186/s12974014-0162-y)

Jayaraman A, Lent-Schochet D \& Pike CJ 2014b Diet-induced obesity and low testosterone increase neuroinflammation and impair neural function. Journal of Neuroinflammation 11 162. (doi:10.1186/s12974014-0162-y)

Kasperk C, Fitzsimmons R, Strong D, Mohan S, Jennings J, Wergedal J \& Baylink D 1990 Studies of the mechanism by which androgens enhance mitogenesis and differentiation in bone cells. Journal of Clinical Endocrinology and Metabolism 71 1322-1329. (doi:10.1210/jcem-71-5-1322)

Kawamura N, Kugimiya F, Oshima Y, Ohba S, Ikeda T, Saito T, Shinoda Y, Kawasaki Y, Ogata N, Hoshi K et al. 2007 Akt1 in osteoblasts and osteoclasts controls bone remodeling. PLOS ONE 2 e1058. (doi:10.1371/ journal.pone.0001058)

Mammi C, Calanchini M, Antelmi A, Cinti F, Rosano GM, Lenzi A, Caprio M \& Fabbri A 2012 Androgens and adipose tissue in males: a complex and reciprocal interplay. International Journal of Endocrinology $\mathbf{2 0 1 2}$ 789653. (doi:10.1155/2012/789653)

Mogri M, Dhindsa S, Quattrin T, Ghanim H \& Dandona P 2013 Testosterone concentrations in young pubertal and post-pubertal obese males. Clinical Endocrinology 78 593-599. (doi:10.1111/cen.12018)

Morakinyo AO, Samuel TA, Adekunbi DA \& Adegoke OA 2015 Niacin improves adiponectin secretion, glucose tolerance and insulin sensitivity in diet-induced obese rats. Egyptian Journal of Basic and Applied Sciences 2 261-267. (doi:10.1016/j.ejbas.2015.08.003)

Ogata N, Chikazu D, Kubota N, Terauchi Y, Tobe K, Azuma Y, Ohta T, Kadowaki T, Nakamura K \& Kawaguchi H 2000 Insulin receptor substrate-1 in osteoblast is indispensable for maintaining bone turnover. Journal of Clinical Investigation 105 935-943. (doi:10.1172/ JCI9017)

Pongkan W, Chattipakorn SC \& Chattipakorn N 2015 Chronic testosterone replacement exerts cardioprotection against cardiac ischemia-reperfusion injury by attenuating mitochondrial dysfunction in testosteronedeprived rats. PLOS ONE 10 e0122503. (doi:10.1371/journal.pone.0122503)

Pramojanee SN, Phimphilai M, Kumphune S, Chattipakorn N \& Chattipakorn SC 2013 Decreased jaw bone density and osteoblastic insulin signaling in a model of obesity. Journal of Dental Research 92 560-565. (doi:10.1177/0022034513485600)

Pratchayasakul W, Kerdphoo S, Petsophonsakul P, Pongchaidecha A, Chattipakorn N \& Chattipakorn SC 2011 Effects of high-fat diet on insulin receptor function in rat hippocampus and the level of neuronal corticosterone. Life Sciences 88 619-627. (doi:10.1016/j.lfs.2011.02.003) 
Pratchayasakul W, Chattipakorn N \& Chattipakorn SC 2014 Estrogen restores brain insulin sensitivity in ovariectomized non-obese rats, but not in ovariectomized obese rats. Metabolism 63 851-859. (doi:10.1016/ j.metabol.2014.03.009)

Pun KK, Lau P \& Ho PW 1989 The characterization, regulation, and function of insulin receptors on osteoblast-like clonal osteosarcoma cell line. Journal of Bone and Mineral Research 4 853-862. (doi:10.1002/ jbmr.5650040610)

Reim NS, Breig B, Stahr K, Eberle J, Hoeflich A, Wolf E \& Erben RG 2008 Cortical bone loss in androgen-deficient aged male rats is mainly caused by increased endocortical bone remodeling. Journal of Bone and Mineral Research 23 694-704. (doi:10.1359/jbmr.080202)

Rosen CJ \& Bouxsein ML 2006 Mechanisms of disease: is osteoporosis the obesity of bone? Nature Clinical Practice. Rheumatology 2 35-43. (doi:10.1038/ncprheum0070)

Saboor Aftab SA, Kumar S \& Barber TM 2013 The role of obesity and type 2 diabetes mellitus in the development of male obesity-associated secondary hypogonadism. Clinical Endocrinology 78 330-337. (doi:10.1111/cen.12092)

Saleem U, Mosley TH \& Kullo IJ 2010 Serum osteocalcin is associated with measures of insulin resistance, adipokine levels, and the presence of metabolic syndrome. Arteriosclerosis, Thrombosis, and Vascular Biology 30 1474-1478. (doi:10.1161/ATVBAHA.110.204859)

Sömjen D, Weisman Y, Harell A, Berger E \& Kaye AM 1989 Direct and sexspecific stimulation by sex steroids of creatine kinase activity and DNA synthesis in rat bone. PNAS 86 3361-3365.

Suntornsaratoon P, Kraidith K, Teerapornpuntakit J, Dorkkam N, Wongdee K, Krishnamra N \& Charoenphandhu N 2014 Pre-suckling calcium supplementation effectively prevents lactationinduced osteopenia in rats. American Journal of Physiology. Endocrinology and Metabolism 306 E177-E188. (doi:10.1152/ajpendo.00556.2013)

Tsujimura A 2013 The relationship between testosterone deficiency and men's health. World Journal of Men's Health 31 126-135. (doi:10.5534/ wjmh.2013.31.2.126)
Tuck SP \& Francis RM 2009 Testosterone, bone and osteoporosis. Frontiers of Hormone Research 37 123-132. (doi:10.1159/000176049)

Vandewalle S, Taes Y, Van Helvoirt M, Debode P, Herregods N, Ernst C, Roef G, Van-Caenegem E, Roggen I, Verhelle F et al. 2013 Bone size and bone strength are increased in obese male adolescents. Journal of Clinical Endocrinology and Metabolism 98 3019-3028. (doi:10.1210/jc. 2012-3914)

Wang C, Jackson G, Jones TH, Matsumoto AM, Nehra A, Perelman MA, Swerdloff RS, Traish A, Zitzmann M \& Cunningham G 2011 Low testosterone associated with obesity and the metabolic syndrome contributes to sexual dysfunction and cardiovascular disease risk in men with type 2 diabetes. Diabetes Care 34 1669-1675. (doi:10.2337/ dc10-2339)

Winzell MS \& Ahren B 2004 The high-fat diet-fed mouse: a model for studying mechanisms and treatment of impaired glucose tolerance and type 2 diabetes. Diabetes 53 S215-S219. (doi:10.2337/diabetes.53. suppl_3.S215)

Wiren KM, Toombs AR, Semirale AA \& Zhang X 2006 Osteoblast and osteocyte apoptosis associated with androgen action in bone: requirement of increased Bax/Bcl-2 ratio. Bone 38 637-651. (doi:10.1016/ j.bone.2005.10.029)

Xia F, Xu X, Zhai H, Meng Y, Zhang H, Du S, Xu H, Wu H \& Lu Y 2013 Castration-induced testosterone deficiency increases fasting glucose associated with hepatic and extra-hepatic insulin resistance in adult male rats. Reproductive Biology and Endocrinology 11 106. (doi:10.1186/ 1477-7827-11-106)

Xiao L, Wang XM, Yang T, Xiong Y, Zhang ZG, Ding J, Xu C \& Xiong CY 2014 Changes of serum osteocalcin, calcium, and potassium in a rat model of type 2 diabetes. Cell Biochemistry and Biophysics 71 437-440. (doi:10.1007/s12013-014-0220-1)

Zhao L-J, Jiang H, Papasian CJ, Maulik D, Drees B, Hamilton J \& Deng H-W 2008 Correlation of obesity and osteoporosis: effect of fat mass on the determination of osteoporosis. Journal of Bone and Mineral Research 23 17-29. (doi:10.1359/jbmr.070813)

Received in final form 9 November 2015

Accepted 13 November 2015
(C) 2016 Society for Endocrinology Printed in Great Britain 